\title{
A UNIFYING APPROACH FOR PROVING HOOK-LENGTH FORMULAS FOR WEIGHTED TREE FAMILIES
}

\author{
MARKUS KUBA AND ALOIS PANHOLZER
}

\begin{abstract}
We propose an expansion technique for weighted tree families, which unifies and extends recent results on hook-length formulas of trees obtained by Han [10], Chen et al. [3], and Yang [19]. Moreover, the approach presented is used to derive new hook-length formulas for tree families, where several hook-functions in the corresponding expansion formulas occur in a natural way. Furthermore we consider families of increasingly labelled trees and show close relations between hook-length formulas for such tree families and corresponding ones for weighted tree families.
\end{abstract}

\section{INTRODUCTION}

Starting with the following remarkable formula given by Postnikov [15]:

$$
\frac{n !}{2^{n}} \sum_{T \in \mathcal{B}(n)} \prod_{v \in T}\left(1+\frac{1}{h_{v}}\right)=(n+1)^{n-1},
$$

where $\mathcal{B}(n)$ denotes the family of binary trees of size $n$, i.e., with $n$ vertices, and $h_{v}$ gives the number of descendants of a node $v$ in a binary tree $T$, various works in the combinatorial literature are devoted to prove and establish such kind of so-called "hook-length formulas". In particular, recently Han [10] developed a very versatile expansion technique for deriving hook-length formulas for partitions and trees and he used his expansion technique to obtain several new formulas for binary trees $[8,9]$.

Han's method was extended by Chen et al. [3] to derive hook-length formulas for other important families of trees, namely $k$-ary trees, planted plane trees and labelled unordered trees, and also for related families of forests. Furthermore, Yang [19] gave extensions of Han's results obtained for binary trees to tree families related to $k$-ary trees and planted plane trees. Moreover, recently Sagan [17] has obtained probabilistic proofs for some of the results of $[3,8,9,10,19]$.

In this work we will extend Han's expansion technique further to treat families of weighted trees, where each node $v$ in the tree gets a weight depending on the out-degree (i.e., the number of children) of $v$. This approach has several favourable features. First it gives a natural way of unifying results of Chen et al. [3], and Yang [19], since all the tree families treated there ( $k$-ary trees, planted plane trees, labelled unordered trees) can be considered as special instances of weighted trees; thus hook-length formulas for these tree families are covered directly by our main result. Second also other interesting tree families, as e.g., Motzkin-trees and labelled cyclic trees, are equivalent to particular weighted tree models and our approach easily leads to hook-length formulas for them. Third the approach presented is flexible enough to cover also situations, where several hook-functions in the corresponding hook-length formulas occur. This naturally appears when considering tree families, where the

Key words and phrases. Hook-length formulas, Weighted trees, Simply generated trees, Increasing trees. The second author was supported by the Austrian Science Foundation FWF, grant S9608-N13. 
weight of a node $v$ also depends on the depth of $v$; note that the depth of a node $v$ in a rooted tree is given by the number of ancestors of $v$ including the node itself, i.e., it measures of nodes on the unique path from $v$ to the root node. Another such situation occurs when considering so-called monotonically labelled tree families. Moreover, we consider so-called increasingly labelled tree families and show that each hook-length formula for a weighted tree family naturally corresponds to a hook-length formula for an increasingly labelled weighted tree family.

We start with a quick review of the terminology. For a rooted tree $T$ the hook-length of a vertex $v \in T$, devoted by $h_{v}:=h(v)$, is the number of descendants of node $v$, where we use the convention that each node $v$ is always considered to be a descendant of itself. For a given weight-function $\rho: \mathbb{N}^{+} \rightarrow \mathbb{C}$ we can associate to any given tree $T$ of some rooted tree family, e.g., $T$ being a binary tree or a planted plane tree, a hook-weight

$$
w_{\text {hook }}(T):=\prod_{v \in T} \rho\left(h_{v}\right) .
$$

In this context the function $\rho$ is called hook-function or hook-weight-function. Han [8] derived the following result for the family of binary trees. Let $F(z)$ be the generating function of the total hook-weights of trees of size $n$, i.e.,

$$
F(z)=\sum_{n \geq 1}\left(\sum_{T \in \mathcal{B}(n)} w_{\text {hook }}(T)\right) z^{n}
$$

then the hook-function $\rho$ satisfies

$$
\rho(n)=\frac{\left[z^{n}\right] F(z)}{\left[z^{n-1}\right](1+F(z))^{2}} .
$$

Here $\left[z^{n}\right]$ denotes the coefficient extraction operator and thus $\left[z^{n}\right] F(z)$ gives the coefficient of $z^{n}$ in the formal power series expansion of $F(z)$. Han used the relation (2) to obtain several hook-length formulas for binary trees, where he used suitable choices of the formal power series $F(z)$. E.g., $F(z)=e^{z}-1$ gives the formula

$$
n ! \sum_{T \in \mathcal{B}(n)} \prod_{v \in T} \frac{1}{h_{v} 2^{h_{v}-1}}=1 .
$$

Choosing $F(z)=\frac{W(2 z)}{2 z}-1$, where $W(z):=\sum_{n \geq 1} \frac{n^{n-1}}{n !} z^{n}$ shall throughout this paper always denote the so-called "tree function" satisfying the functional equation $W(z)=z e^{W(z)}$, proves formula (1) obtained by Postnikov.

As mentioned before, by using Han's method, the results (3) for binary trees were extended by Chen et al. [3] and by Yang [19] to other tree families, including $k$-ary trees, labelled unordered trees and planted plane trees. These extensions were used to derive new hooklength formulas for the before mentioned tree families. Moreover, Chen et al. [3] derived hook-length formulas for forests of trees by suitable extensions of (3). In the next section we will consider families of weighted trees and derive a relation generalizing (3), which unifies the formulas given in $[3,19]$ and allows to derive hook-length formulas for further tree families as exemplified by results for Motzkin-trees and labelled cyclic trees. In Section 3 we show extensions of the approach leading to hook-length formulas including several hook-functions. In Section 4 we consider hook-length formulas for increasing tree families.

Besides the notation introduced at other places, we denote, for a rooted tree $T$, by $r:=r(T)$ the root-node of $T$ and by $|T|$ the size of $T$, i.e., the number vertices of $T$, whereas, for any node $v \in T$, the out-degree (i.e., the number of children) of $v$ is denoted by $\operatorname{deg}(v)$. 
Furthermore, $\operatorname{EN}(T)$ denotes the set of end-nodes (= leaves) of a rooted tree $T$, i.e., all vertices $v$ satisfying $\operatorname{deg}(v)=0$ or equivalently $h_{v}=1$; analogous for the set of end-nodes $\operatorname{EN}(F)$ of a forest $F$. Moreover, for any given tree family $\mathcal{S}$, we use $\mathcal{S}(n)$ to denote the tree family containing all trees of $\mathcal{S}$ that have size $n$.

\section{Weighted tree FAMILIES AND HOOK-LENGTH FORMULAS}

2.1. An expansion formula for weighted tree families. One of our main motivations to consider weighted tree families is to obtain hook-length formulas for important and wellstudied combinatorial objects called simply generated tree families [13], sometimes also denoted as simple varieties of trees [4]. The basic objects considered in this context are ordered trees, also called planted plane trees, i.e., rooted trees, where to each node $v$ there is attached a (possibly empty) sequence of child-nodes (thus the left-to-right order of the children is important). Throughout this paper we denote by $\mathcal{O}$ the family of ordered trees. Different simply generated tree models are then obtained by considering weighted ordered trees, where each node $v$ in an ordered tree $T \in \mathcal{O}$ gets a certain "degree-weight factor" depending on the out-degree of $v$, i.e., the number of children of $v$, and the degree-weight of the tree $T$ is defined as the product of the degree-weight factors of all of its nodes. It is well-known that many important (unweighted) combinatorial tree families are equivalent to such weighted tree models, where the degree-weights are chosen in a specific way; see [4] and examples below. We want to add that in a probabilistic context it is very natural to consider weighted trees and, in particular, that in probability theory the combinatorial models of simply generated trees are known as Galton-Watson-trees arising from certain branching processes, see, e.g., [1]. In this context the meaning of the degree-weight of a node is closely related to the probability that, during the branching process, a specific node gets a certain amount of children. We note that for simply generated trees one always assumes that the degree-weight of a node is a non-negative real number. However, for our purpose we can omit this restriction and we will even allow that the degree-weights of the nodes are arbitrary complex numbers; the obtained hook-length formulas for weighted trees still hold.

Formally, a family $\mathcal{T}$ of weighted ordered trees can be defined as follows. A sequence of complex numbers $\left(\varphi_{j}\right)_{j \geq 0}$ is used to define the multiplicative degree-weight $\varphi_{j}$ of a node with out-degree $j$. The degree-weight $w_{\operatorname{deg}}(T)$ of any ordered tree $T \in \mathcal{O}$ is defined by $w_{\operatorname{deg}}(T):=\prod_{v \in T} \varphi_{\operatorname{deg}(v)}$, i.e., as the product of all degree-weight factors of the vertices $v$ of $T$; recall that $\operatorname{deg}(v)$ denotes the out-degree of a node $v$. In order to avoid degenerate cases we always assume that $\varphi_{0} \neq 0$. The family $\mathcal{T}$ consists then of all ordered trees $T$ (or equivalently of all ordered trees $T$ with $w_{\operatorname{deg}}(T) \neq 0$ ) together with their degree-weights $w_{\operatorname{deg}}(T)$, i.e., one might think of pairs $\left(T, w_{\operatorname{deg}}(T)\right)$. According to a previous definition $\mathcal{T}(n)$ denotes the family of weighted ordered trees of size $n$, i.e., all pairs $\left(T, w_{\operatorname{deg}}(T)\right)$, with $|T|=n$.

It follows from the definition that, for a given degree-weight sequence $\left(\varphi_{j}\right)_{j \geq 0}$, the formal power series $T(z):=\sum_{n>1} T_{n} z^{n}$, where the quantity $T_{n}:=\sum_{T \in \mathcal{O}(n)} w_{\operatorname{deg}}(T)$ measures the total degree-weights of ordered trees of size $n$, satisfies the functional equation

$$
T(z)=z \varphi(T(z)) ;
$$

the degree-weight generating function $\varphi(t)$ is there given by $\varphi(t):=\sum_{j \geq 0} \varphi_{j} t^{j}$.

Next we describe the expansion technique for weighted ordered tree families to obtain hooklength formulas for such tree models. Given a hook-weight function (or hook-function, for short) $\rho: \mathbb{N}^{+} \rightarrow \mathbb{C}$ and a degree-weight sequence $\left(\varphi_{j}\right)_{j \geq 0}$ (or alternatively its degree-weight generating function $\varphi(t)=\sum_{j \geq 0} \varphi_{j} t^{j}$ ) we can associate to any ordered tree $T \in \mathcal{O}$ a weight 
$w(T):=w_{\operatorname{deg}}(T) \cdot w_{\text {hook }}(T)$, which is defined as the product of the hook-weight $w_{\text {hook }}(T):=$ $\prod_{v \in T} \rho\left(h_{v}\right)$ and the degree-weight $w_{\operatorname{deg}}(T):=\prod_{v \in T} \varphi_{\operatorname{deg}(v)}$. Note that this definition of the weight $w(T)$ of a tree $T$ generalizes the weights associated to families of simply generated trees, which, of course, can be recovered by using the constant hook-function $\rho(n)=1$, for all $n \in \mathbb{N}$. The name expansion technique or expansion formula for the following theorem reflects the fact that we start with an expansion of the power series (i.e., the generating function) associated to the sum of the weights of all trees of a given size, which we use to derive the hook-weight function involved.

Theorem 1. Given a family $\mathcal{T}$ of weighted ordered trees associated to a degree-weight generating function $\varphi(t)$, let $F(z)$ be the generating function of the total weights of trees of size n, i.e.,

$$
F(z)=\sum_{n \geq 1}\left(\sum_{T \in \mathcal{O}(n)} w(T)\right) z^{n}=\sum_{n \geq 1}\left(\sum_{T \in \mathcal{O}(n)} w_{d e g}(T) \cdot \prod_{v \in T} \rho\left(h_{v}\right)\right) z^{n} .
$$

Then the hook-function $\rho$ satisfies

$$
\rho(n)=\frac{\left[z^{n}\right] F(z)}{\left[z^{n-1}\right] \varphi(F(z))}, \quad n \geq 1 .
$$

Proof. Let $F_{n}=\left[z^{n}\right] F(z)$. By definition we have

$$
F_{n}=\sum_{T \in \mathcal{O}(n)} w(T)=\sum_{T \in \mathcal{O}(n)} w_{\operatorname{deg}}(T) \cdot w_{\text {hook }}(T) .
$$

Following Han [8], see also Chen et al. [3] and Yang [19], we use the so-called top-bottom decomposition of a tree, i.e., the decomposition of a rooted tree into the root-node $r$ and the subtrees attached to the root. We consider a tree $T$ of size $n \geq 2$ and assume that the root $r$ of $T$ has out-degree $j \geq 1$. Then the root-node gives a degree-weight factor $\varphi_{\operatorname{deg}(r)}=\varphi_{j}$ and a hook-weight factor $\rho\left(h_{r}\right)=\rho(n)$, whereas the subtrees $T_{1}, \ldots, T_{j}$ dangling from the root have certain degree-weights $w_{\operatorname{deg}}\left(T_{\ell}\right)$ and hook-weights $w_{\text {hook }}\left(T_{\ell}\right)$, for $1 \leq \ell \leq j$. Due to the multiplicative definition of the weight $w(T)$ of the tree $T$, we obtain thus the following factorization:

$$
w(T)=w_{\operatorname{deg}}(T) \cdot w_{\text {hook }}(T)=\varphi_{j} \cdot \rho(n) \prod_{\ell=1}^{j}\left(w_{\operatorname{deg}}\left(T_{\ell}\right) \cdot w_{\text {hook }}\left(T_{\ell}\right)\right)=\varphi_{j} \cdot \rho(n) \prod_{\ell=1}^{j} w\left(T_{\ell}\right) .
$$

By considering all possible ways of building a tree of size $n \geq 2$ we get

$$
\begin{aligned}
F_{n} & =\sum_{T \in \mathcal{O}(n)} w_{\mathrm{deg}}(T) \cdot w_{\text {hook }}(T)=\rho(n) \sum_{j \geq 1} \varphi_{j} \sum_{\substack{n_{1}+\cdots+n_{j}=n-1, T_{1} \in \mathcal{O}\left(n_{1}\right), \ldots, T_{j} \in \mathcal{O}\left(n_{j}\right) \\
n_{1}, \ldots, n_{j} \geq 1}} \prod_{\ell=1}^{j} w\left(T_{\ell}\right) \\
& =\rho(n) \sum_{j \geq 1} \varphi_{j} \sum_{\substack{n_{1}+\cdots+n_{j}=n-1, n_{1}, \ldots, n_{j} \geq 1}}\left(\sum_{T_{1} \in \mathcal{O}\left(n_{1}\right)} w\left(T_{1}\right)\right) \cdot\left(\sum_{T_{2} \in \mathcal{O}\left(n_{2}\right)} w\left(T_{2}\right)\right) \cdots\left(\sum_{T_{j} \in \mathcal{O}\left(n_{j}\right)} w\left(T_{j}\right)\right) \\
& =\rho(n) \sum_{j \geq 1} \varphi_{j} \sum_{\substack{n_{1}+\cdots+n_{j}=n-1, n_{1}, \ldots, n_{j} \geq 1}} \prod_{\ell=1}^{j} F_{n_{\ell}}=\rho(n)\left[z^{n-1}\right] \varphi(F(z)) .
\end{aligned}
$$

It only remains to check the initial case $n=1$. In accordance to the definition $F_{1}=\varphi_{0} \cdot \rho(1)$, we get for $n=1$ the equation $\rho(1)=\frac{\left[z^{1}\right] F(z)}{\left[z^{0}\right] \varphi(F(z))}=\frac{F_{1}}{\varphi_{0}}$, which shows that the theorem holds for all $n \geq 1$. 


\subsection{Applications to important tree models.}

2.2.1. Unlabelled tree families. As already mentioned previously, various important combinatorial tree families are equivalent to families of weighted ordered trees with specific degreeweights. In particular this situation occurs if, for a rooted tree family $\mathcal{S}$, there exists a weighted ordered tree family $\mathcal{T}$ associated to a degree-weight generating function $\varphi(t)$, such that for each ordered tree $T \in \mathcal{O}$ the following relation holds:

$$
w_{\mathrm{deg}}(T)=\sum_{\substack{T^{\prime} \in \mathcal{S}: \\ \operatorname{shape}\left(T^{\prime}\right)=T}} 1,
$$

where shape $\left(T^{\prime}\right)=T$, if there exists an isomorphism from the rooted tree $T^{\prime}$ to the ordered tree $T$ that also preserves the linear order of the children of the nodes. Let us now consider an arbitrary hook-function $\rho$. Since the hook-weight is the same for all isomorphic trees, one further gets, for each $T \in \mathcal{O}$, the relation:

$$
w(T)=w_{\text {hook }}(T) \cdot w_{\mathrm{deg}}(T)=\sum_{T^{\prime} \in \mathcal{S}: \operatorname{shape}\left(T^{\prime}\right)=T} w_{\text {hook }}(T)=\sum_{T^{\prime} \in \mathcal{S}: \operatorname{shape}\left(T^{\prime}\right)=T} w_{\text {hook }}\left(T^{\prime}\right),
$$

which implies

$$
\sum_{T \in \mathcal{O}(n)} w(T)=\sum_{T \in \mathcal{O}(n)} \sum_{T^{\prime} \in \mathcal{S}(n): \operatorname{shape}\left(T^{\prime}\right)=T} w_{\text {hook }}\left(T^{\prime}\right)=\sum_{T^{\prime} \in \mathcal{S}(n)} w_{\text {hook }}\left(T^{\prime}\right) .
$$

Thus, since $F(z)=\sum_{n \geq 1} \sum_{T \in \mathcal{O}(n)} w(T) z^{n}=\sum_{n \geq 1} \sum_{T^{\prime} \in \mathcal{S}(n)} w_{\text {hook }}\left(T^{\prime}\right) z^{n}$, relation (6) simply says that the hook-expansion formula (5) in Theorem 1 for the weighted tree family $\mathcal{T}$ associated to the degree-weight generating function $\varphi(t)$ exactly corresponds to a hookexpansion formula for the family $\mathcal{S}$.

Example 1. Of course, the family $\mathcal{O}$ of ordered trees (= planted plane trees) is contained in our weighted ordered tree model by using the degree-weights $\varphi_{j}=1$, for $j \geq 0$, i.e., by using the degree-weight generating function $\varphi(t)=\frac{1}{1-t}$. The family $\mathcal{B}$ of binary trees, i.e., trees where each node is either a leaf or it might have a left and/or a right child, is equivalent to the weighted ordered tree model by choosing $\varphi_{0}=1, \varphi_{1}=2, \varphi_{2}=1$, and $\varphi_{j}=0$, for $j \geq 3$, i.e., $\varphi(t)=(1+t)^{2}$. More generally, the family $\mathcal{B}_{k}$ of $k$-ary trees, i.e., trees where each node has exactly $k$ positions, where a child might be attached or not, is equivalent to the family of weighted ordered trees associated to $\varphi_{j}=\left(\begin{array}{l}k \\ j\end{array}\right)$, for $j \geq 0$, and thus $\varphi(t)=(1+t)^{k}$. A generalization of the before-mentioned tree models are so-called binomial families of trees, which again can be modelled by weighted ordered trees, now associated to degree-weight generating functions $\varphi(t)=(1+s t)^{\alpha}$, with certain real parameters $s$ and $\alpha$. Thus Theorem 1 for weighted ordered trees covers the expansion techniques for the above tree families and therefore unifies the corresponding results stated in $[3,10,19]$.

Example 2. We further illustrate Theorem 1 by deriving hook-length formulas for another important tree family, namely the family $\mathcal{M}$ of Motzkin-trees, which, in this context, has not been considered so far. Motzkin-trees, also called unary-binary trees, are rooted trees, where each node has 0,1 , or 2 children. They are enumerated by the Motzkin-numbers and there are close relations to Motzkin-paths (see, e.g., [4]). It is immediate from the definition that $\mathcal{M}$ is equivalent to the family of weighted ordered trees associated to $\varphi_{j}=1$, for $0 \leq j \leq 2$, and $\varphi_{j}=0$, for $j \geq 3$, and thus $\varphi(t)=1+t+t^{2}$. Next we want to apply Theorem 1 with 
$\varphi(t)=1+t+t^{2}$ to the function $F(z)=\frac{1}{2}\left(\frac{W(2 z)}{2 z}-1\right)$. Using the Lagrange-Bürmann inversion formula, see, e.g., [4], one easily obtains

$$
\begin{gathered}
{\left[z^{n}\right] F(z)=\left[z^{n}\right] \frac{1}{2}\left(\frac{W(2 z)}{2 z}-1\right)=\frac{2^{n}(n+1)^{n}}{2(n+1) !}} \\
{\left[z^{n-1}\right] \varphi(F(z))=\left[z^{n-1}\right] \frac{3}{4}+\frac{1}{4} \frac{W(2 z)^{2}}{(2 z)^{2}}= \begin{cases}\frac{2^{n}(n+1)^{n-1}}{4(n+1)(n-1) !}, & \text { for } n \geq 2, \\
1, & \text { for } n=1 .\end{cases} }
\end{gathered}
$$

Formula (5) leads thus to the hook-function

$$
\rho(n)= \begin{cases}2\left(1+\frac{1}{n}\right), & \text { for } n \geq 2 \\ 1, & \text { for } n=1\end{cases}
$$

This leads to the following hook-length formula for Motzkin-trees, which can be considered as an analogon to formula (1) obtained by Postnikov for binary trees:

$$
n ! \sum_{T \in \mathcal{M}(n)} \frac{1}{2^{|\mathrm{EN}(T)|-1}} \prod_{v \in T \backslash \mathrm{EN}(T)}\left(1+\frac{1}{h_{v}}\right)=(n+1)^{n-1} .
$$

Moreover, with $\varphi(t)=1+t+t^{2}$ and by using the function $F(z)=\frac{1}{2}\left(e^{z}-1\right)$, an application of (5) immediately leads to the hook-function

$$
\rho(n)= \begin{cases}\frac{1}{n 2^{n-2}}, & \text { for } n \geq 2 \\ \frac{1}{2}, & \text { for } n=1\end{cases}
$$

which shows the following analogue for Motzkin-trees of Han's binary tree-formula (4):

$$
n ! \sum_{T \in \mathcal{M}(n)} \frac{1}{2^{|\mathrm{EN}(T)|-1}} \prod_{v \in T \backslash \mathrm{EN}(T)} \frac{1}{h_{v} 2^{h_{v}-2}}=1 .
$$

2.2.2. Labelled tree families. The model of weighted ordered trees is flexible enough to describe also various important labelled tree models, where we suppose that trees of size $n$ are labelled with distinct integers of the set $\{1,2, \ldots, n\}$. We consider now the following situation. We assume that, for a labelled rooted tree family $\mathcal{S}$, there exists a weighted (unlabelled) ordered tree family $\mathcal{T}$ associated to a degree-weight generating function $\varphi(t)$, such that, for each ordered tree $T \in \mathcal{O}$, the following relation holds:

$$
n ! \cdot w_{\operatorname{deg}}(T)=\sum_{\substack{T^{\prime} \in \mathcal{S} \\ \operatorname{shape}\left(T^{\prime}\right)=T}} 1 .
$$

Let us now consider an arbitrary hook-function $\rho$. Then one further obtains, for each $T \in \mathcal{O}$, the relation:

which implies

$$
n ! \cdot w(T)=\sum_{T^{\prime} \in \mathcal{S}: \operatorname{shape}\left(T^{\prime}\right)=T} w_{\text {hook }}\left(T^{\prime}\right),
$$

$$
\sum_{T \in \mathcal{O}(n)} w(T)=\sum_{T \in \mathcal{O}(n)} \sum_{T^{\prime} \in \mathcal{S}(n): \operatorname{shape}\left(T^{\prime}\right)=T} \frac{w_{\mathrm{hook}}\left(T^{\prime}\right)}{n !}=\sum_{T^{\prime} \in \mathcal{S}(n)} \frac{w_{\mathrm{hook}}\left(T^{\prime}\right)}{n !} .
$$

Thus, since $F(z)=\sum_{n \geq 1} \sum_{T \in \mathcal{O}(n)} w(T) z^{n}=\sum_{n \geq 1} \sum_{T^{\prime} \in \mathcal{S}(n)} w_{\text {hook }}\left(T^{\prime}\right) \frac{z^{n}}{n !}$, the hook-expansion formula (5) in Theorem 1 for the weighted tree family $\mathcal{T}$ associated to the degree-weight generating function $\varphi(t)$ exactly corresponds to a hook-expansion formula for the labelled tree family $\mathcal{S}$. 
Example 3. The family $\mathcal{U}$ of labelled unordered trees, i.e., labelled rooted trees, where to each node $v$ in a tree there is attached a set of children (i.e., the left-to-right order of the children is not taken into account), can be modelled by the weighted ordered tree family $\mathcal{T}$ with degree-weights $\varphi_{j}=1 / j$ !, for $j \geq 0$, i.e., $\varphi(t)=e^{t}$. Thus Theorem 1 covers the expansion technique for the family of labelled unordered trees, which appears in [3].

Example 4. As another example we consider the family $\mathcal{C}$ of so-called labelled cyclic trees (also called labelled mobile trees) considered in $[2,4,5]$. Each node $v$ in such a labelled tree is either an end-node or there is attached a cycle of children, i.e., one might assume that the children of each node are arranged via circular shifts such that the child with smallest label is always the leftmost child. This family can be modelled by the weighted ordered tree family $\mathcal{T}$ with degree-weights $\varphi_{0}=1$ and $\varphi_{j}=1 / j$, for $j \geq 1$, i.e., $\varphi(t)=1+\log \left(\frac{1}{1-t}\right)$.

We apply now Theorem 1 with $\varphi(t)=1+\log \left(\frac{1}{1-t}\right)$ to the function $F(z)=1-e^{-W(z)}$. Using the Lagrange-Bürmann inversion formula one easily obtains, for $n \geq 1$ :

$$
\left[z^{n}\right] F(z)=\frac{(n-1)^{n-1}}{n !}, \quad\left[z^{n-1}\right] \varphi(F(z))= \begin{cases}\frac{(n-1)^{n-2}}{(n-1) !}, & \text { for } n \geq 2 \\ 1, & \text { for } n=1\end{cases}
$$

Formula (5) leads thus to the hook-function

$$
\rho(n)= \begin{cases}1-\frac{1}{n}, & \text { for } n \geq 2 \\ 1, & \text { for } n=1\end{cases}
$$

This gives the following hook-length formula for labelled mobile trees, which also can be considered as an analogue to formula (1):

$$
\sum_{T \in \mathcal{C}(n)} \prod_{v \in T \backslash \mathrm{EN}(T)}\left(1-\frac{1}{h_{v}}\right)=(n-1)^{n-1} .
$$

2.3. Formulas for forests of weighted trees. In [3] hook-length formulas for so-called plane forests, i.e., sequences of ordered trees, and forests of labelled trees, i.e., sets of labelled unordered trees, have been derived. In the following we consider certain forests of weighted ordered trees, for which we will extend the expansion technique. By doing this one regains the known expansion formulas for plane forests and forests of labelled trees, since they appear as particular instances.

We assume that, for a family $\mathcal{T}$ of weighted ordered trees associated to a degree-weight sequence $\left(\varphi_{j}\right)_{j \geq 0}$, a hook-weight function $\rho^{+}: \mathbb{N} \rightarrow \mathbb{C}$ is given. Thus, as described in Subsection 2.1, we can define the weight $w(T)$ of an ordered tree $T \in \mathcal{O}$ as $w(T)=w_{\operatorname{deg}}(T) \cdot w_{\text {hook }}(T)$, with $w_{\operatorname{deg}}(T)=\prod_{v \in T} \varphi_{\operatorname{deg}(v)}$ and $w_{\text {hook }}(T)=\prod_{v \in T} \rho\left(h_{v}\right)$. Next we define weighted ordered forests. Our basic objects are finite sequences $F=\left(T_{1}, \ldots, T_{k}\right)$ of ordered trees $T_{\ell} \in \mathcal{O}$, $1 \leq \ell \leq k$, where we allow the empty sequence $F=\epsilon$; we call such an object an ordered forest. The family of ordered forests (also called plane forests) will be denoted by $\mathcal{O}_{F}$. The size $|F|$ of a forest $F=\left(T_{1}, \ldots, T_{k}\right)$ is defined via $|F|:=\left|T_{1}\right|+\cdots+\left|T_{k}\right| ;$ additionally we set $|\epsilon|=0$. To each ordered forest $F \in \mathcal{O}_{F}$ we define a weight $w(F)$ as follows:

$$
w(F):=\varphi_{k} \prod_{\ell=1}^{k} w\left(T_{\ell}\right), \quad \text { if } F=\left(T_{1}, \ldots, T_{k}\right), \quad \text { and } \quad w(\epsilon)=\varphi_{0} .
$$

The family $\mathcal{F}$ of weighted ordered forests consists then of all ordered forests $F$ together with the weights $w(F)$. If we consider the generating function $F(z):=\sum_{n \geq 1} \sum_{T \in \mathcal{O}(n)} w(T) z^{n}$ of the total weights of trees of size $n$, and the generating function $G(z):=\sum_{n \geq 0} \sum_{F \in \mathcal{O}_{F}(n)} w(F) z^{n}$ 
of the total weights of forests of size $n$, then from the above definition of the weights the following relation between these generating functions easily follows:

$$
G(z)=\varphi(F(z)) .
$$

In the following theorem we describe the expansion formula for weighted ordered forests.

Theorem 2. Given a family $\mathcal{F}$ of weighted ordered forests associated to a degree-weight generating function $\varphi(t)=\sum_{j \geq 0} \varphi_{j} t^{j}$, with $\varphi_{0} \neq 0$ and $\varphi_{1} \neq 0$, let $G(z)$ be the generating function of the total weights of forests of size $n$, i.e.,

$$
G(z)=\sum_{n \geq 0} \sum_{F \in \mathcal{O}_{F}(n)} w(F) z^{n} .
$$

Then the hook-weight function $\rho$ satisfies

$$
\rho(n)=\frac{\left[z^{n}\right] \tilde{\varphi}^{[-1]}\left(G(z)-\varphi_{0}\right)}{\left[z^{n-1}\right] G(z)}, \quad n \geq 1,
$$

where $\tilde{\varphi}(t):=\varphi(t)-\varphi_{0}$, and $\tilde{\varphi}^{[-1]}(t)$ denotes the inverse function of $\tilde{\varphi}(t)$, i.e., $\tilde{\varphi}\left(\tilde{\varphi}^{[-1]}(t)\right)=$ $\tilde{\varphi}\left(\tilde{\varphi}^{[-1]}(t)\right)=t$.

Proof. Due to $\varphi_{1} \neq 0$ there exist numbers $\psi_{j}, j \geq 1$, such that $\tilde{\varphi}^{[-1]}(t)=\sum_{j \geq 1} \psi_{j} t^{j}$, i.e., there exists a formal power series representing the inverse function. From (11) one gets the relation $G(z)=\varphi_{0}+\tilde{\varphi}(F(z))$ between the generating functions of weighted trees and forests, which implies

$$
F(z)=\sum_{n \geq 1} \sum_{T \in \mathcal{O}(n)} w(T) z^{n}=\tilde{\varphi}^{[-1]}\left(G(z)-\varphi_{0}\right) .
$$

Hence, when applying Theorem 1 to $F(z)$ and using relation (13), we obtain

$$
\begin{aligned}
\rho(n) & =\frac{\left[z^{n}\right] \tilde{\varphi}^{[-1]}\left(G(z)-\varphi_{0}\right)}{\left[z^{n-1}\right] \varphi\left(\tilde{\varphi}^{[-1]}\left(G(z)-\varphi_{0}\right)\right)}=\frac{\left[z^{n}\right] \tilde{\varphi}^{[-1]}\left(G(z)-\varphi_{0}\right)}{\left[z^{n-1}\right]\left(\varphi_{0}+\tilde{\varphi}\left(\tilde{\varphi}^{[-1]}\left(G(z)-\varphi_{0}\right)\right)\right)} \\
& =\frac{\left[z^{n}\right] \tilde{\varphi}^{[-1]}\left(G(z)-\varphi_{0}\right)}{\left[z^{n-1}\right] G(z)}, \quad n \geq 1 .
\end{aligned}
$$

Example 5. The theorems in [3] concerning expansion formulas for plane forests $O_{F}$ and forests of labelled trees $U_{F}$ can be obtained from Theorem 2 by considering the degree-weight generating functions $\varphi(t)=1 /(1-t)$ (for plane forests), such that

$$
\tilde{\varphi}^{[-1]}(t)=1-\frac{1}{1+t}, \quad \rho(n)=\frac{\left[z^{n}\right] \tilde{\varphi}^{[-1]}(G(z)-1)}{\left[z^{n-1}\right] G(z)}=\frac{-\left[z^{n}\right](G(z))^{-1}}{\left[z^{n-1}\right] G(z)},
$$

and $\varphi(t)=e^{t}$ (for forests of labelled trees), such that

$$
\tilde{\varphi}^{[-1]}(t)=\log (1+t), \quad \rho(n)=\frac{\left[z^{n}\right] \tilde{\varphi}^{[-1]}(G(z)-1)}{\left[z^{n-1}\right] G(z)}=\frac{\left[z^{n}\right] \log G(z)}{\left[z^{n-1}\right] G(z)} .
$$

Example 6. We consider the family of labelled cyclic forests $\mathcal{C}_{F}$, i.e., forests which consist of sequences of labelled cyclic trees, where cyclic rearrangements of a particular sequence of trees are considered to be equivalent; one might also say that the family $\mathcal{C}_{F}$ consists of 
cycles of labelled cyclic trees. Thus they can be described by weighted ordered forests with a degree-weight generating function $\varphi(t)=1+\log \left(\frac{1}{1-t}\right)=1+\sum_{j \geq 1} \frac{1}{j} t^{j}$ and one gets:

$$
\tilde{\varphi}^{[-1]}(t)=1-e^{-t}, \quad \rho(n)=\frac{\left[z^{n}\right] \tilde{\varphi}^{[-1]}(G(z)-1)}{\left[z^{n-1}\right] G(z)}=-\frac{\left[z^{n}\right] e^{-G(z)+1}}{\left[z^{n-1}\right] G(z)}, \quad n \geq 1 .
$$

When considering the function $G(z)=W(z)+1$, then one obtains, after applying the Lagrange-Bürmann inversion formula, the following hook-length formula for the family $\mathcal{C}_{F}$ of labelled cyclic forests:

$$
\sum_{F \in \mathcal{C}_{F}(n)} \prod_{v \in F \backslash \operatorname{EN}(F)}\left(1-\frac{1}{h_{v}}\right)=n^{n-1} .
$$

2.4. Combining hook-length formulas for partitions and trees. Theorem 1 can be used in conjunction with existing hook-length formulas for integer partitions (see, e.g., [10]), to derive new hook-length formulas, which might be more involved. We recall some basic notation; for general definitions concerning the class $\mathcal{P}$ of integer partitions we refer to [18] and for definitions in the context of hook-length formulas to [10]. A partition $\lambda$ of an integer $n$ is a sequence of positive integers $\lambda=\left(\lambda_{1}, \lambda_{2}, \ldots, \lambda_{\ell}\right)$ such that $\lambda_{1} \geq \lambda_{2} \geq \cdots \geq \lambda_{\ell}>0$ and $|\lambda|:=\lambda_{1}+\cdots+\lambda_{\ell}=n$; the family of partitions of $n$ is denoted by $\mathcal{P}(n)$. For each box $v$ in the Ferrers diagram of a partition $\lambda$ one defines the hook-length $h_{v}=h(v)$ of $v$ as the number of boxes $u$, such that either $u=v$, or $u$ lies in the same column as $v$ and above $v$, or $u$ lies in the same row as $v$ and to the right of $v$. The hook-length multiset of $\lambda$, denoted by $\mathcal{H}(\lambda)$, is the multiset of all hook-lengths of $\lambda$. In the following we give one particular example.

Example 7. We consider a family $\mathcal{T}$ of weighted ordered trees associated to a degree-weight sequence $\left(\varphi_{j}\right)_{j \geq 0}$. Let $f(z)$ be the Nekrasov-Okounkov hook-length function for partitions (see, e.g., [10]):

$$
f(z)=\sum_{\lambda \in \mathcal{P}} z^{|\lambda|} \prod_{h \in \mathcal{H}(\lambda)}\left(1-\frac{x}{h^{2}}\right)=\prod_{k \geq 1}\left(1-z^{k}\right)^{x-1}, \quad x \in \mathbb{C} .
$$

Note that extracting coefficients of $f^{j}(z)$ can be done easily by substituting $x$ by $j x-(j-1)$ in the previous formula, which gives

$$
\left[z^{n}\right] f^{j}(z)=\left[z^{n}\right] \prod_{k \geq 1}\left(1-z^{k}\right)^{j x-(j-1)-1}=\sum_{\lambda \in \mathcal{P}(n)} \prod_{h \in \mathcal{H}(\lambda)}\left(1-\frac{j x-(j-1)}{h^{2}}\right), \quad j \in \mathbb{N} .
$$

We define now $F(z):=f(z)-1$ and apply Theorem 1 to this function. This yields

$$
\rho(n)=\frac{\left[z^{n}\right] F(z)}{\left[z^{n-1}\right] \varphi(F(z))}=\frac{\left[z^{n}\right] f(z)}{\left[z^{n-1}\right] \sum_{\ell=0}^{n-1} \varphi_{\ell} \cdot(f(z)-1)^{\ell}}=\frac{\left[z^{n}\right] f(z)}{\left[z^{n-1}\right] \sum_{j=0}^{n-1} f^{j}(z) \sum_{\ell=j}^{n-1}\left(\begin{array}{l}
\ell \\
j
\end{array}\right)(-1)^{\ell-j} \varphi_{\ell}} .
$$

Thus, we obtain the following formula combining hook-length formulas for the family $\mathcal{T}$ of weighted ordered trees and the family $\mathcal{P}$ of integer partitions:

$$
\begin{array}{r}
\sum_{T \in \mathcal{O}(n)} \prod_{v \in T}\left(\frac{\varphi_{\operatorname{deg}(v)} \cdot \sum_{\lambda \in \mathcal{P}\left(h_{v}\right)} \prod_{h \in \mathcal{H}(\lambda)}\left(1-\frac{x}{h^{2}}\right)}{\sum_{j=0}^{h_{v}-1} \sum_{\ell=j}^{h_{v}-1}\left(\begin{array}{l}
\ell \\
j
\end{array}\right)(-1)^{\ell-j} \varphi_{\ell} \cdot \sum_{\lambda \in \mathcal{P}\left(h_{v}-1\right)} \prod_{h \in \mathcal{H}(\lambda)}\left(1-\frac{j x-(j-1)}{h^{2}}\right)}\right) \\
=\sum_{\lambda \in \mathcal{P}(n)} \prod_{h \in \mathcal{H}(\lambda)}\left(1-\frac{x}{h^{2}}\right),
\end{array}
$$


where the denominator in the product on the left-hand side has to be interpreted as $\varphi_{0}$ whenever $h_{v}=1$.

\section{Generalizations to several hook-Functions}

Han's expansion technique can also be applied to various other tree families, which are not covered directly by the concept of weighted ordered trees as introduced in Section 2. In the following we highlight the versatility of this method by studying several extensions of Theorem 1, which contain more than one hook-weight-function.

3.1. Hook-length formulas with hook-functions depending on the depth of nodes. We consider now weighted ordered trees, where the degree-weights of the nodes are depending also on the depth of the nodes. In particular we assume that there are two different degree-weight sequences $\left(\varphi_{j}^{[1]}\right)_{j \geq 0}$ and $\left(\varphi_{j}^{[2]}\right)_{j \geq 0}$ (or, equivalently, two different degree-weight generating functions $\varphi^{[1]}(t)=\sum_{j \geq 0} \varphi_{j}^{[1]} t^{j}$ and $\left.\varphi^{[2]}(t)=\sum_{j \geq 0} \varphi_{j}^{[2]} t^{j}\right)$ and each node $v$ of out-degree $j$ in an ordered tree gets, depending whether the depth of $v$ is an odd or an even number, the degree-weight factor $\varphi_{j}^{[1]}$ or $\varphi_{j}^{[2]}$, respectively. We note that such kind of weighted ordered trees arise naturally in various contexts (see, e.g., [12] for an example in connection with bipartite planar maps) and they are known in the probabilistic literature as certain two-type Galton-Watson trees, where nodes of type $A$ only bear children of type $B$ and vice versa. For such tree families $\mathcal{T}$ we consider two different hook-weight-functions $\rho^{[1]}$ : $\mathbb{N}^{+} \rightarrow \mathbb{C}$ and $\rho^{[2]}: \mathbb{N}^{+} \rightarrow \mathbb{C}$ and each node $v$ in the tree will get, depending on the parity of the depth of $v$, a hook-weight factor $\rho^{[1]}\left(h_{v}\right)$ or $\rho^{[2]}\left(h_{v}\right)$, respectively. Moreover, for a given ordered tree $T \in \mathcal{O}$ we define two different weights $w^{[1]}(T)$ and $w^{[2]}(T)$ as follows:

$$
\begin{aligned}
& w^{[1]}(T):=w_{\text {hook }}^{[1]}(T) \cdot w_{\operatorname{deg}}^{[1]}(T)=\prod_{\substack{v \in T: \\
\operatorname{depth}(v) \equiv 1}}\left(\varphi_{\operatorname{deg}(v)}^{[1]} \rho^{[1]}\left(h_{v}\right)\right) \cdot \prod_{\substack{\bmod 2 \\
\operatorname{depth}(v) \equiv 0}}\left(\varphi_{\operatorname{deg}(v)}^{[2]} \rho^{[2]}\left(h_{v}\right)\right), \\
& w^{[2]}(T):=w_{\text {hook }}^{[2]}(T) \cdot w_{\operatorname{deg}}^{[2]}(T)=\prod_{\substack{v \in T: \\
\operatorname{depth}(v) \equiv 1}}\left(\varphi_{\operatorname{deg}(v)}^{[2]} \rho^{[2]}\left(h_{v}\right)\right) \cdot \prod_{\substack{v \in T: \\
\bmod 2}}\left(\varphi_{\operatorname{deg}(v)}^{[1]} \rho^{[1]}\left(h_{v}\right)\right) .
\end{aligned}
$$

The following theorem describes the expansion technique for such kind of tree families.

Theorem 3. Given a weighted tree family $\mathcal{T}$ associated to degree-weight generating functions $\varphi^{[1]}(t)$ and $\varphi^{[2]}(t)$, let $F^{[1]}(z)$ and $F^{[2]}(z)$ be the corresponding generating functions of the total weights of trees of size $n$, i.e.,

$$
F^{[1]}(z)=\sum_{n \geq 1}\left(\sum_{T \in \mathcal{O}(n)} w^{[1]}(T)\right) z^{n}, \quad F^{[2]}(z)=\sum_{n \geq 1}\left(\sum_{T \in \mathcal{O}(n)} w^{[2]}(T)\right) z^{n} .
$$

Then the hook-weight functions $\rho^{[1]}$ and $\rho^{[2]}$ satisfy, for $n \geq 1$, the relations

$$
\rho^{[1]}(n)=\frac{\left[z^{n}\right] F^{[1]}(z)}{\left[z^{n-1}\right] \varphi^{[1]}\left(F^{[2]}(z)\right)}, \quad \rho^{[2]}(n)=\frac{\left[z^{n}\right] F^{[2]}(z)}{\left[z^{n-1}\right] \varphi^{[2]}\left(F^{[1]}(z)\right)} .
$$

Proof. We only outline the main steps of the proof, since it is similar to the proof of Theorem 1. Let $F_{n}^{[1]}=\left[z^{n}\right] F^{[1]}(z)$. By definition we have

$$
F_{n}^{[1]}=\sum_{T \in \mathcal{O}(n)} w^{[1]}(T)=\sum_{T \in \mathcal{O}(n)} w_{\mathrm{deg}}^{[1]}(T) w_{\mathrm{hook}}^{[1]}(T) .
$$


Let us assume that the root $r$ of a given size- $n$ tree $T$ has out-degree $j \geq 1$. Then the weight $w^{[1]}(T)$ factorizes into a degree-weight factor $\varphi_{d(r)}^{[1]}=\varphi_{j}^{[1]}$, a hook-weight factor $\rho^{[1]}\left(h_{r}\right)=$ $\rho^{[1]}(n)$, and factors corresponding to the $j$ non-empty subtrees $T_{\ell}, 1 \leq \ell \leq j$, dangling from the root. Note that, since the depths of all nodes contained in the $j$ non-empty subtrees $T_{\ell}$, $1 \leq \ell \leq j$, are in $T$ increased by one, this leads, according to the definition of $w^{[1]}(T)$ and $w^{[2]}(T)$, to a change of the corresponding weight-functions for the $j$ subtrees and we obtain:

$$
w^{[1]}(T)=w_{\text {deg }}^{[1]}(T) w_{\text {hook }}^{[1]}(T)=\varphi_{j}^{[1]} \cdot \rho^{[1]}(n) \cdot \prod_{\ell=1}^{j}\left(w_{\text {deg }}^{[2]}\left(T_{\ell}\right) w_{\text {hook }}^{[2]}\left(T_{\ell}\right)\right) .
$$

By considering all possible ways to build a tree of size $n$ we get

$$
\begin{aligned}
F_{n}^{[1]} & =\sum_{T \in \mathcal{O}(n)} w_{\text {deg }}^{[1]}(T) w_{\text {hook }}^{[1]}(T)=\rho^{[1]}(n) \sum_{j \geq 1} \varphi_{j}^{[1]} \sum_{\substack{n_{1}+\ldots+n_{j}=n-1, T_{1} \in \mathcal{O}\left(n_{1}\right), \ldots, T_{j} \in \mathcal{O}\left(n_{j}\right) \\
n_{1}, \ldots, n_{j} \geq 1}} \prod_{\ell=1}^{j} w^{[2]}\left(T_{\ell}\right) \\
& =\rho^{[1]}(n) \sum_{j \geq 1} \varphi_{j}^{[1]} \sum_{\substack{n_{1}+\cdots+n_{j}=n-1, n_{1}, \ldots, n_{j} \geq 1}} \prod_{\ell=1}^{j} F_{n_{\ell}}^{[2]}=\rho^{[1]}(n)\left[z^{n-1}\right] \varphi^{[1]}\left(F^{[2]}(z)\right) .
\end{aligned}
$$

One easily checks that the formula is also valid for the initial case $n=1$. The opposite case concerning $F^{[2]}(z)$ is proven similarly.

We remark that for constant hook-weight functions $\rho^{[1]}=\rho^{[2]}=1$ we obtain the simple relations

$$
F^{[1]}(z)=z \varphi^{[1]}\left(F^{[2]}(z)\right), \quad F^{[2]}(z)=z \varphi^{[2]}\left(F^{[1]}(z)\right) .
$$

We illustrate Theorem 3 by the following example, which generalizes Theorem 2.4 of Chen et al. [3]; it appears as the special instance $k=\ell, a=b$ and $u=w$ in the example below.

Example 8. We consider the degree-weight generating functions $\varphi^{[1]}(t)=(1+t)^{\ell}$ and $\varphi^{[2]}(t)=(1+t)^{k}$, with $\ell, k \in \mathbb{N}$. Let $g_{1}(z)$ and $g_{2}(z)$ be implicitly defined by the functional equations

$$
g_{1}(z)=(a-k) z\left(1+g_{1}(z)\right)^{\frac{k(a-1)}{a-k}}, \quad g_{2}(z)=(b-\ell) z\left(1+g_{2}(z)\right)^{\frac{\ell(b-1)}{b-\ell}}
$$

and let

$$
F^{[1]}(z):=\left(1+g_{1}(z)\right)^{\frac{u a}{a-k}}-1, \quad F^{[2]}(z):=\left(1+g_{2}(z)\right)^{\frac{w b}{b-\ell}}-1 .
$$

Applying the Lagrange-Bürmann inversion formula yields:

$$
\left[z^{n}\right] F^{[1]}(z)=\frac{u a}{n !} \prod_{i=1}^{n-1}(u a+k(a-1) n-i(a-k)), \quad\left[z^{n}\right] F^{[2]}(z)=\frac{w b}{n !} \prod_{i=1}^{n-1}(w b+\ell(b-1) n-i(b-\ell)) .
$$

Furthermore, it holds $\left[z^{n-1}\right] \varphi^{[1]}\left(F^{[2]}(z)\right)=\left[z^{n-1}\right]\left(1+g_{2}(z)\right)^{\frac{w b \ell}{b-\ell}}$, and $\left[z^{n-1}\right] \varphi^{[2]}\left(F^{[1]}(z)\right)=$ $\left[z^{n-1}\right]\left(1+g_{1}(z)\right)^{\frac{u a k}{a-k}}$; thus extracting coefficients can be done easily by using the relations (19) and substituting $w$ by $w \ell$ and $u$ by $u k$, respectively. An application of Theorem 3 shows then the following hook-length formula for this family $\mathcal{T}$ of weighted ordered trees:

$$
\sum_{T \in \mathcal{O}(n)}\left[\prod_{\substack{v \in T: \\
\operatorname{depth}(v) \equiv 1(2)}}\left(\begin{array}{c}
\ell \\
\operatorname{deg}(v)
\end{array}\right) \frac{u a}{w b \ell h_{v}} \frac{\prod_{i=1}^{h_{v}-1}\left(u a+k(a-1) h_{v}-i(a-k)\right)}{\prod_{i=1}^{h_{v}-2}\left(w b \ell+\ell(b-1)\left(h_{v}-1\right)-i(b-\ell)\right)}\right.
$$




$$
\begin{aligned}
& \left.\times \prod_{\substack{v \in T: \\
\operatorname{depth}(v) \equiv 0(2)}}\left(\begin{array}{c}
k \\
\operatorname{deg}(v)
\end{array}\right) \frac{w b}{u a k h_{v}} \frac{\prod_{i=1}^{h_{v}-1}\left(w b+\ell(b-1) h_{v}-i(b-\ell)\right)}{\prod_{i=1}^{h_{v}-2}\left(u a k+k(a-1)\left(h_{v}-1\right)-i(a-k)\right)}\right] \\
& =\frac{u a}{n !} \prod_{i=1}^{n-1}(u a+k(a-1) n-i(a-k)) .
\end{aligned}
$$

Note that, for $k=\ell, a=b$ and $u=w$, this weighted ordered trees have an interpretation as $k$-ary trees, which exactly leads to the corresponding result stated in [3].

We remark that Theorem 3 can be generalized further in various ways. For example, one may consider $k \geq 1$ different degree-weight generating functions $\varphi^{[\ell]}(t), 1 \leq \ell \leq k$, and also $k$ different hook-weight functions $\rho^{[\ell]}: \mathbb{N}^{+} \rightarrow \mathbb{C}, 1 \leq \ell \leq k$, leading to $k$ different weight functions $w^{[\ell]}(T)$ :

$$
w^{[\ell]}(T):=w_{\text {hook }}^{[\ell]}(T) \cdot w_{\operatorname{deg}}^{[\ell]}(T)=\prod_{i=1}^{k}\left(\prod_{\substack{v \in T: \\ \operatorname{depth}(v) \equiv i-1+\ell \bmod k}} \varphi_{\operatorname{deg}(v)}^{[i]} \rho^{[i]}\left(h_{v}\right)\right), \quad 1 \leq \ell \leq k .
$$

We remark that such weighted ordered tree models are related to certain multitype GaltonWatson trees. Similar to the proof of Theorem 3 one can show the following theorem (whose proof is thus omitted) describing the expansion technique for such weighted ordered trees.

Theorem 4. Let the functions $F^{[\ell]}(z), 1 \leq \ell \leq k$, defined as follows:

$$
F^{[\ell]}(z)=\sum_{n \geq 1}\left(\sum_{T \in \mathcal{O}(n)} w^{[\ell]}(T)\right) z^{n}
$$

and additionally set $F^{[k+1]}(z):=F^{[1]}(z)$. Then it holds

$$
\rho^{[\ell]}(n)=\frac{\left[z^{n}\right] F^{[\ell]}(z)}{\left[z^{n-1}\right] \varphi^{[\ell]}\left(F^{[\ell+1]}(z)\right)}, \quad 1 \leq \ell \leq k .
$$

Note that Theorems 1, 3 correspond to the cases $k=1,2$ in Theorem 4, respectively.

3.2. Generalized monotonically labelled trees. We consider now families of weighted ordered trees, where the nodes are labelled monotonically decreasing by elements of the set $\{1, \ldots, s\}$, i.e., the sequence of labels lying on any path from the root to an arbitrary node in the tree is weakly monotone decreasing. Such tree structures generalize the notion of simply generated trees, see Section 2, and were introduced ${ }^{1}$ by Prodinger and Urbanek [16], and studied further in $[7,11]$. For a given ordered tree $T \in \mathcal{O}$ let $\mathcal{L}^{[s]}(T)$ denote the set of monotone decreasing labellings of the nodes of $T$ with elements of the set $\{1,2, \ldots, s\}$; each labelling $L \in \mathcal{L}^{[s]}(T)$ maps the vertices $v \in T$ to the integers $\{1,2, \ldots, s\}$ in a suitable way. We introduce now an extension of the model of weighted ordered trees, where the degree-weight factor of a node $v$ also depends on the label of $v$, i.e., if node $v$ of out-degree $j$ is labelled by $\ell, L(v)=\ell$, then the degree-weight factor of $v$ shall be given by $\varphi_{j}^{[\ell]}$. Thus,

\footnotetext{
${ }^{1}$ Originally such tree families were introduced by assuming that the nodes along any path starting at the root of a tree are forming a weakly monotone increasing sequence; however, in the present context it is more convenient to consider decreasing labellings.
} 
for given degree-weight generating functions $\varphi^{[\ell]}(t)=\sum_{j \geq 0} \varphi_{j}^{[\ell]} t^{j}, 1 \leq \ell \leq s$, we define the degree-weight $w_{\mathrm{deg}}(T, L)$ of a tree $T$ with labelling $L \in \mathcal{L}^{[s]}(T)$ as follows:

$$
w_{\operatorname{deg}}(T, L):=\prod_{v \in T} \varphi_{\operatorname{deg}(v)}^{[L(v)]}
$$

Let $\mathcal{T}_{s}$ denote the family of such weighted ordered trees, where the nodes are labelled monotonically decreasing by elements of $\{1, \ldots, s\}$. It is not difficult to show (see, e.g., $[11,16]$ ) that the generating functions $T_{s}(z):=\sum_{n \geq 1} \sum_{T \in \mathcal{O}(n)} \sum_{L \in \mathcal{L}^{[s]}(T)} w_{\operatorname{deg}}(T, L) z^{n}$ associated to the families $\mathcal{T}_{s}$ satisfy the following system of functional equations:

$$
T_{1}(z)=z \varphi^{[1]}\left(T_{1}(z)\right), \quad T_{\ell}(z)=z \varphi^{[\ell]}\left(T_{\ell}(z)\right)+T_{\ell-1}(z), \quad 2 \leq \ell \leq s .
$$

In order to show hook-length formulas for these tree families it is natural to consider $s$ hookweight functions $\rho^{[\ell]}, 1 \leq \ell \leq s$, and define the weight $w(T, L)$ of an ordered tree $T \in \mathcal{O}$ with labelling $L \in \mathcal{L}^{[s]}(T)$ as follows (i.e., also the hook-weight factor of a node $v$ depends on the label of $v$ ):

$$
w(T, L):=w_{\mathrm{deg}}(T, L) w_{\mathrm{hook}}(T, L), \quad w_{\mathrm{deg}}(T, L):=\prod_{v \in T} \varphi_{\operatorname{deg}(v)}^{[L(v)]}, \quad w_{\mathrm{hook}}(T, L):=\prod_{v \in T} \rho^{[L(v)]}\left(h_{v}\right) .
$$

The following theorem describes then the expansion technique for the families $\mathcal{T}_{s}$ of monotonically labelled weighted ordered trees.

Theorem 5. Given a monotonically labelled weighted tree family $\mathcal{T}_{s}$ associated to degreeweight generating functions $\varphi^{[\ell]}(t), 1 \leq \ell \leq s$, let $F_{\ell}(z), 1 \leq \ell \leq s$, be the generating functions of the total weights of monotonically labelled trees of $\mathcal{T}_{\ell}$ of size $n$, i.e.,

$$
F_{\ell}(z)=\sum_{n \geq 1}\left(\sum_{T \in \mathcal{O}(n)} \sum_{L \in \mathcal{L}^{[\ell]}(T)} w(T, L)\right) z^{n}, \quad 1 \leq \ell \leq s .
$$

Then the hook-functions $\rho^{[\ell]}, 1 \leq \ell \leq s$, satisfy

$$
\rho^{[1]}(n)=\frac{\left[z^{n}\right] F_{1}(z)}{\left[z^{n-1}\right] \varphi^{[1]}\left(F_{1}(z)\right)}, \quad \rho^{[\ell]}(n)=\frac{\left[z^{n}\right] F_{\ell}(z)-\left[z^{n}\right] F_{\ell-1}(z)}{\left[z^{n-1}\right] \varphi^{[\ell]}\left(F_{\ell}(z)\right)}, \quad 2 \leq \ell \leq s .
$$

Proof. Since the proof uses the same ideas as used for the previous results we just sketch it. Consider the function $F_{\ell}(z)$, with $2 \leq \ell \leq s$. By definition we have

$$
\begin{aligned}
{\left[z^{n}\right] F_{\ell}(z) } & =\sum_{T \in \mathcal{O}(n)} \sum_{L \in \mathcal{L}^{[\ell]}(T)} w(T, L) \\
& =\sum_{T \in \mathcal{O}(n)} \sum_{L \in \mathcal{L}^{[\ell]}(T): L(r)=\ell} w(T, L)+\sum_{T \in \mathcal{O}(n)} \sum_{L \in \mathcal{L}^{[\ell]}(T): L(r) \leq \ell-1} w(T, L) \\
& =\sum_{T \in \mathcal{O}(n)} \sum_{L \in \mathcal{L}^{[\ell]}(T): L(r)=\ell} w(T, L)+\left[z^{n}\right] F_{\ell-1}(z) .
\end{aligned}
$$

Let us now assume that the root $r$ of a given size- $n$ tree $T$ with labelling $L$ has out-degree $j \geq 1$ and is labelled by $\ell$. Then the weight $w(T, L)$ factorizes into a degree-weight factor $\varphi_{j}^{[\ell]}$, a hook-weight factor $\rho^{[\ell]}(n)$, and factors $w\left(T_{i}, L_{i}\right)$ corresponding to the $j$ subtrees $T_{i}$ with 
labellings $L_{i}, 1 \leq i \leq j$, dangling from the root. By considering all possible ways to build a monotonically decreasing labelled tree of size $n$, such that the root is labelled by $\ell$, we get

$$
\begin{aligned}
& \sum_{T \in \mathcal{O}(n)} \sum_{L \in \mathcal{L}^{[\ell]}(T): L(r)=\ell} w(T, L)=\rho^{[\ell]}(n) \sum_{j \geq 1} \varphi_{j}^{[\ell]} \sum_{\substack{n_{1}+\cdots+n_{j}=n-1 \\
n_{1}, \ldots, n_{j} \geq 1}} \prod_{i=1}^{j} \sum_{T_{i} \in \mathcal{T}\left(n_{i}\right)} \sum_{L_{i} \in \mathcal{L}^{[\ell]}\left(T_{i}\right)} w\left(T_{i}, L_{i}\right) \\
& =\rho^{[\ell]}(n) \sum_{j \geq 1} \varphi_{j}^{[\ell]} \sum_{\substack{n_{1}+\cdots+n_{j}=n-1, i=1 \\
n_{1}, \ldots, n_{j} \geq 1}} \prod_{i=1}^{j}\left[z^{n_{i}}\right] F_{\ell}(z)=\rho^{[\ell]}(n)\left[z^{n-1}\right] \varphi^{[\ell]}\left(F_{\ell}(z)\right) .
\end{aligned}
$$

Combining these results shows the theorem for $2 \leq \ell \leq s$; the case $\ell=1$ corresponds to unlabelled weighted ordered trees and has thus been proven in Theorem 1 .

Example 9. We illustrate Theorem 5 by considering monotonically labelled trees with degree-weight generating functions $\varphi^{[\ell]}(t)=(1+t)^{\ell}, 1 \leq \ell \leq s$, i.e., each node of label $\ell$ might be considered as an " $\ell$-ary node". We will apply the expansion technique to functions $F_{\ell}(z)=e^{\frac{z}{\ell}}-1$. Extracting coefficients according to (22) easily gives

$$
\rho^{[1]}(n)=\frac{1}{n}, \quad \rho^{[\ell]}(n)=\left(\frac{1}{\ell^{n}}-\frac{1}{(\ell-1)^{n}}\right) \frac{1}{n}, \quad 2 \leq \ell \leq s .
$$

This shows the following hook-length formula for the family $\mathcal{T}_{s}$ :

$$
\sum_{T \in \mathcal{O}(n)} \sum_{L \in \mathcal{L}^{[s]}(T)}\left(\prod_{v \in T}\left(\begin{array}{c}
L(v) \\
\operatorname{deg}(v)
\end{array}\right) \frac{1}{h_{v}}\right) \cdot \prod_{v \in T: L(v)>1}\left(\frac{1}{L(v)^{h_{v}}}-\frac{1}{(L(v)-1)^{h_{v}}}\right)=\frac{1}{s^{n} n !} .
$$

3.3. Trees with a special treatment of the root. Some combinatorial tree families (as, e.g., plane trees as considered in [5], and so-called non-crossing trees [14]) are not equivalent to weighted ordered trees as introduced in Section 2, since the root of any such tree has to be treated in a separate way. However, one can easily extend the concept of weighted ordered trees to cover also such situations.

We assume that there are two degree-weight generating functions, namely $\varphi^{[r]}(t)=\sum_{j \geq 1} \varphi_{j}^{[r]} t^{j}$ for the root-node and $\varphi^{[q]}(t)=\sum_{j \geq 1} \varphi_{j}^{[q]} t^{j}$ for the non-root nodes, and two hook-weight functions $\rho^{[r]}$ and $\rho^{[q]}$ for the root-node and non-root-nodes, respectively. For a given ordered tree $T \in \mathcal{O}$ we define two different degree-weights $w_{\text {deg }}^{[1]}(T), w_{\text {deg }}^{[2]}(T)$ and hook-weights $w_{\text {hook }}^{[1]}(T)$, $w_{\text {hook }}^{[2]}(T)$ as follows:

$$
\begin{array}{rlrl}
w_{\mathrm{deg}}^{[1]}(T) & :=\varphi_{\operatorname{deg}(r)}^{[r]} \prod_{v \in T: v \neq r} \varphi_{\operatorname{deg}(v)}^{[q]}, & w_{\text {hook }}^{[1]}(T):=\rho_{h_{r}}^{[r]} \prod_{v \in T: v \neq r} \rho^{[q]}\left(h_{v}\right), \\
w_{\operatorname{deg}}^{[2]}(T):=\prod_{v \in T} \varphi_{\operatorname{deg}(v)}^{[q]}, & w_{\text {hook }}^{[2]}(T):=\prod_{v \in T} \rho^{[q]}\left(h_{v}\right) .
\end{array}
$$

A straightforward extension of the proof of Theorem 1 shows then the following expansion formula for such tree families; thus we may omit the proof.

Theorem 6. For given degree-weight generating functions $\varphi^{[r]}(t)$ and $\varphi^{[q]}(t)$ let the functions $F(z)$ and $G(z)$ defined as follows:

$$
F(z)=\sum_{n \geq 1}\left(\sum_{T \in \mathcal{O}(n)} w_{\text {deg }}^{[1]}(T) w_{\text {hook }}^{[1]}(T)\right) z^{n}, \quad G(z)=\sum_{n \geq 1}\left(\sum_{T \in \mathcal{O}(n)} w_{\text {deg }}^{[2]}(T) w_{\text {hook }}^{[2]}(T)\right) z^{n} .
$$


Then the hook-weight functions $\rho^{[r]}$ and $\rho^{[q]}$ satisfy the relations

$$
\rho^{[r]}(n)=\frac{\left[z^{n}\right] F(z)}{\left[z^{n-1}\right] \varphi^{[r]}(G(z))}, \quad \rho^{[q]}(n)=\frac{\left[z^{n}\right] G(z)}{\left[z^{n-1}\right] \varphi^{[q]}(G(z))}, \quad n \geq 1 .
$$

Example 10. A non-crossing tree is a tree whose vertices $1,2, \ldots, n$ are located at the corners of a regular $n$-gon and whose edges are non-crossing straight line segments; the tree is considered as being rooted at vertex 1 . It is known that the family $\mathcal{N}$ of non-crossing trees is equivalent to weighted ordered trees associated to degree-weight generating functions $\varphi^{[r]}(t)=\frac{1}{1-t}$ and $\varphi^{[q]}(t)=\frac{1}{(1-t)^{2}}$ for the root-node and non-root-nodes, respectively (see, e.g., $[14]$ and references therein). When applying Theorem 6 to the functions $F(z)=G(z)=1-e^{z}$ one easily obtains the following hook-length formula for the family $\mathcal{N}$ non-crossing trees:

$$
(-1)^{n-1}(n-1) ! \sum_{T \in \mathcal{N}(n)} \prod_{v \in T \backslash r(T)} \frac{(-1)^{h_{v}}}{h_{v} 2^{h_{v}-1}}=1 .
$$

\section{INCREASING TREES AND HOOK-LENGTH FORMULAS}

4.1. The hook-expansion formula for weighted increasing trees. Increasing trees are labelled trees, where the nodes of a tree of size $n$ are labelled by distinct integers of the set $\{1, \ldots, n\}$ in such a way that each sequence of labels along any path starting at the root is increasing. Several hook-length formulas appearing in the literature have an interpretation in terms of increasing trees (see, e.g., [3, 6]). In the following we consider families of increasingly labelled weighted ordered trees (weighted increasing trees for short), and show that hooklength formulas for weighted ordered trees have a natural counterpart in hook-length formulas for weighted increasing trees and vice versa.

Let us denote by $\hat{\mathcal{O}}$ the family of ordered increasing trees, i.e., increasingly labelled ordered trees. We assume that a degree-weight sequence $\left(\varphi_{j}\right)_{j \geq 0}$, with $\varphi_{0} \neq 0$, is given. Then, analogous to weighted ordered trees, a family $\hat{\mathcal{T}}$ of weighted increasing trees can be defined in the following way. The degree-weight $w_{\operatorname{deg}}(\hat{T})$ of any ordered increasing tree $\hat{T} \in \hat{\mathcal{O}}$ is defined by $w_{\operatorname{deg}}(\hat{T}):=\prod_{v \in \hat{T}} \varphi_{\operatorname{deg}(v)}$. The family $\hat{\mathcal{T}}$ consists then of all ordered increasing trees $\hat{T}$ together with their degree-weights $w_{\mathrm{deg}}(\hat{T})$. For a given degree-weight sequence $\left(\varphi_{j}\right)_{j \geq 0}$ with a degree-weight generating function $\varphi(t):=\sum_{j \geq 0} \varphi_{j} t^{j}$, we define now the total degreeweights by $T_{n}:=\sum_{\hat{T} \in \hat{\mathcal{O}}(n)} w_{\operatorname{deg}}(\hat{T})$. It can be shown easily that the corresponding exponential generating function $T(z):=\sum_{n \geq 1} T_{n} \frac{z^{n}}{n !}$ satisfies the first order differential equation $T^{\prime}(z)=$ $\varphi(T(z))$, with $T(0)=0$.

Given a family $\hat{\mathcal{T}}$ of weighted increasing trees associated to a degree-weight generating function $\varphi(t)$ we consider now a hook-weight-function $\hat{\rho}: \mathbb{N}^{+} \rightarrow \mathbb{C}$. Completely analogous to Section 2 one can define the hook-weight $\hat{w}_{\text {hook }}(\hat{T})$ and the weight $w(\hat{T})$ of an increasing ordered tree $\hat{T}$ via $\hat{w}_{\text {hook }}(\hat{T}):=\prod_{v \in \hat{T}} \hat{\rho}\left(h_{v}\right)$, and $w(\hat{T}):=w_{\operatorname{deg}}(\hat{T}) \cdot \hat{w}_{\text {hook }}(\hat{T})$. Using the decomposition of an ordered increasing tree into the root node and its subtrees as celebrated earlier several times one can show the following expansion formula for weighted increasing trees; however, we will skip this proof, since below we will give an easy argument leading to it, too.

Theorem 7. Given a family $\hat{\mathcal{T}}$ of weighted increasing trees associated to a degree-weight generating function $\varphi(t)$, let $\hat{F}(z)$ be the exponential generating function of the total weights 
of trees of size n, i.e.,

$$
\hat{F}(z)=\sum_{n \geq 1}\left(\sum_{\hat{T} \in \hat{\mathcal{O}}(n)} w(\hat{T})\right) z^{n} .
$$

Then the hook-function $\hat{\rho}$ satisfies

$$
\hat{\rho}(n)=\frac{\left[z^{n-1}\right] \hat{F}^{\prime}(z)}{\left[z^{n-1}\right] \varphi(\hat{F}(z))}=\frac{n\left[z^{n}\right] \hat{F}(z)}{\left[z^{n-1}\right] \varphi(\hat{F}(z))}, \quad n \geq 1 .
$$

4.2. Connections between weighted ordered trees and weighted increasing trees. Alternatively, a family $\hat{\mathcal{T}}$ of weighted increasing trees can be defined also by starting with a family $\mathcal{T}$ of weighted ordered trees associated to a degree-weight generating function $\varphi(t)$ and considering, for each ordered tree $T \in \mathcal{O}$, all increasing labellings of it. Given an ordered tree $T$ we denote by $\mathcal{L}_{I}(T)$ the set of increasing labellings of $T$, i.e, labellings of the nodes of the tree $T$ with distinct integers from $\{1,2, \ldots,|T|\}$ such that the sequence of labels along any path starting at the root is increasing. Thus the members of a family $\hat{\mathcal{T}}$ of weighted increasing trees could be considered as pairs $(T, L)$, with $T$ an ordered tree and $L \in \mathcal{L}_{I}(T)$ an increasing labelling, together with their degree-weights $w_{\operatorname{deg}}(T)$ defined as for weighted ordered trees.

It is well-known (see, e.g., [6]) and can be shown easily by induction, that for any ordered tree $T \in \mathcal{O}(n)$ the number $\left|\mathcal{L}_{I}(T)\right|$ of increasing labellings of $T$ is given as follows:

$$
\left|\mathcal{L}_{I}(T)\right|=\frac{n !}{\prod_{v \in T} h_{v}} .
$$

Now, we consider a family $\mathcal{T}$ of weighted ordered trees, and also the corresponding family $\hat{\mathcal{T}}$ of weighted increasing trees, both associated to the degree-weight generating function $\varphi(t)$. Furthermore, we assume that for the family $\mathcal{T}$ a hook-weight function $\rho: \mathbb{N}^{+} \rightarrow \mathbb{C}$ and for the family $\hat{\mathcal{T}}$ a hook-weight function $\hat{\rho}: \mathbb{N}^{+} \rightarrow \mathbb{C}$ is given. The following lemma shows that each hook-length formula for a weighted ordered tree family naturally corresponds to a hook-length formula for the corresponding weighted increasing tree family and vice versa.

Lemma 8. If the hook-weight functions $\hat{\rho}$ and $\rho$ of the families $\hat{\mathcal{T}}$ and $\mathcal{T}$, respectively, satisfy the relation

$$
\hat{\rho}(n)=n \rho(n),
$$

then the following relation between the total weights of weighted increasing trees $\hat{T} \in \hat{\mathcal{O}}(n)$ and weighted ordered trees $T \in \mathcal{O}(n)$ holds:

$$
\sum_{\hat{T} \in \hat{\mathcal{O}}(n)} w_{\text {deg }}(\hat{T}) \cdot \hat{w}_{h o o k}(\hat{T})=n ! \sum_{T \in \mathcal{O}(n)} w_{\text {deg }}(T) \cdot w_{h o o k}(T) .
$$

Proof.

$$
\begin{aligned}
& \sum_{\hat{T} \in \hat{\mathcal{O}}(n)} w_{\mathrm{deg}}(\hat{T}) \cdot \hat{w}_{\mathrm{hook}}(\hat{T})=\sum_{T \in \mathcal{O}(n)} \sum_{L \in \mathcal{L}_{I}(T)} w_{\mathrm{deg}}(T) \cdot \hat{w}_{\mathrm{hook}}(T) \\
= & \sum_{T \in \mathcal{O}(n)} w_{\operatorname{deg}}(T) \hat{w}_{\mathrm{hook}}(T) \cdot\left|\mathcal{L}_{I}(T)\right|=\sum_{T \in \mathcal{O}(n)} w_{\mathrm{deg}}(T) \prod_{v \in T} \hat{\rho}\left(h_{v}\right) \cdot \prod_{v \in T} \frac{n !}{\prod_{v \in T} h_{v}} \\
= & n ! \sum_{T \in \mathcal{O}(n)} w_{\mathrm{deg}}(T) \prod_{v \in T} \rho\left(h_{v}\right)=n ! \sum_{T \in \mathcal{O}(n)} w_{\operatorname{deg}}(T) \cdot w_{\mathrm{hook}}(T) .
\end{aligned}
$$


When assuming that $\hat{\rho}(n)=n \rho(n)$ and defining the functions

$$
F(z)=\sum_{n \geq 1} \sum_{T \in \mathcal{O}(n)} w_{\operatorname{deg}}(T) w_{\text {hook }}(T) z^{n}, \quad \hat{F}(z)=\sum_{n \geq 1} \sum_{\hat{T} \in \hat{\mathcal{O}}(n)} w_{\operatorname{deg}}(\hat{T}) \hat{w}_{\text {hook }}(\hat{T}) \frac{z^{n}}{n !},
$$

we get $F(z)=\hat{F}(z)$ by applying Lemma 8; thus an application of Theorem 1 also shows Theorem 7 .

Example 11. Let us denote by $\hat{\mathcal{B}}$ the family of increasingly labelled binary trees; it is well-known that this tree-model is equivalent to so-called binary search trees generated by permutations (see, e.g., [4]), which appear frequently in theoretical computer science. Using (27) we obtain from Han's formula (4) the following hook-length formula for increasingly labelled binary trees (and for binary search trees):

$$
\sum_{\hat{T} \in \hat{\mathcal{B}}(n)} \prod_{v \in \hat{T}} \frac{1}{2^{h_{v}-1}}=1 \text {. }
$$

We want to add that, when considering relation (27) with hook-weight-functions $\rho(n)=\frac{1}{n}$ and $\hat{\rho}(n)=n \rho(n)=1$, respectively, then $\hat{w}_{\text {hook }}(\hat{T})=1$ for any tree $\hat{T} \in \hat{\mathcal{O}}$ and the left-hand side of (27) simply counts the total degree-weights of weighted increasing trees in $\hat{\mathcal{T}}$ of size $n$. Since various weighted ordered tree models are equivalent to important combinatorial tree families $\mathcal{S}$ as already mentioned previously, in such cases the left-hand side can be interpreted simply as the number of increasingly labelled trees of $\mathcal{S}$ of size $n$. We add that such a hook-function $\rho(n)=\frac{1}{n}$ corresponds to the relation $F^{\prime}(z)=\varphi(F(z))$ and $F(0)=0$, when considering the corresponding generating functions $F(z)$ and $\hat{F}(z)$.

Example 12. We consider exemplarily the case $\varphi(t)=1 /(1-t)^{\alpha}, \alpha>0$. The function $F(z)$ satisfying $F^{\prime}(z)=\varphi(F(z))$ and $F(0)=0$ has the solution $F(z)=1-(1-(\alpha+1) z)^{1 /(\alpha+1)}$. Hence, we obtain $\left[z^{n}\right] F(z)=\sum_{T \in \mathcal{O}(n)} w_{\operatorname{deg}}(T) \cdot w_{\text {hook }}(T)=(\alpha+1)^{n-1}(n-1) !\left(\begin{array}{c}n-1-\frac{1}{\alpha+1} \\ n-1\end{array}\right)$ and thus the hook-length formula

$$
\sum_{T \in \mathcal{O}(n)} \prod_{v \in T}\left(\begin{array}{c}
\alpha-1+\operatorname{deg}(v) \\
\operatorname{deg}(v)
\end{array}\right) \cdot \frac{n !}{\prod_{v \in T} h_{v}}=(\alpha+1)^{n-1}(n-1) !\left(\begin{array}{c}
n-1-\frac{1}{\alpha+1} \\
n-1
\end{array}\right),
$$

where the right-hand side has the interpretation of the total degree-weights of increasingly labelled trees of $\mathcal{T}$ of size $n$. The case $\alpha=1$ corresponds to increasingly labelled ordered trees (so-called plane recursive trees) and appears in [3].

\section{ACKNOWLEDGEMENTS}

The authors thank W. Y. C. Chen for encouraging remarks.

\section{REFERENCES}

[1] D. Aldous, The continuum random tree. An overview. Stochastic Analysis, 23-70, London Mathematical Society Lecture Notes Series, 167, Cambridge University Press, 1991.

[2] F. Bergeron, G. Labelle and P. Leroux, Combinatorial species and tree-like structures. Cambridge University Press, Cambridge, 1998.

[3] W. Y. C. Chen, O. X. Q. Gao, and P. L. Guo, Hook length formulas for trees by Han's expansion. Electronic Journal of Combinatorics, 16, \#R62, 2009.

[4] P. Flajolet and R. Sedgewick, Analytic combinatorics. Cambridge University Press, Cambridge, 2009.

[5] I. Gessel, B. Sagan and Y.-N. Yeh, Enumeration of trees by inversions. Journal of Graph Theory, 19, 435-459, 1995. 
[6] I. Gessel and S. Seo, A refinement of Cayley's formula for trees. Electronic Journal of Combinatorics, 11(2), \#R27, 2006.

[7] B. Gittenberger and A. Panholzer, Some results for monotonically labelled simply generated trees. Discrete Mathematics and Theoretical Computer Science, Proceedings AD, 173-180, 2005.

[8] G.-N. Han, New hook length formulas for binary trees. Combinatorica, 30, 253-256, 2010.

[9] G.-N. Han, Yet another generalization of Postnikovs hook length formula for binary trees. SIAM Journal on Discrete Mathematics, 23, 661-664, 2009.

[10] G.-N. Han, Discovering hook length formulas by an expansion technique. Electronic Journal of Combinatorics, 15, \#R133, 2008.

[11] P. Kirschenhofer, On the average shape of monotonically labelled tree structures. Discrete Applied Mathematics, 7, 161-181, 1984.

[12] J.-F. Marckert and G. Miermont, Invariance principles of random bipartite planar maps. The Annals of Probability, 35, 1642-1705, 2007.

[13] A. Meir and J. W. Moon, On the altitude of nodes in random trees. Canadian Journal of Mathematics, 30, 997-1015, 1978.

[14] A. Panholzer and H. Prodinger, Bijections for ternary trees and non-crossing trees. Discrete Mathematics, 250, 181-195, 2002.

[15] A. Postnikov, Permutohedra, associahedra, and beyond. International Mathematics Research Notices, 6 , 1026-1106, 2009.

[16] H. Prodinger and F. J. Urbanek. On monotone functions of tree structures. Discrete Applied Mathematics, 5, 223-239, 1983.

[17] B. E. Sagan, Probabilistic proofs of hook length formulas involving trees. Séminaire Lotharingien de Combinatoire, 61A, Article B61Ab, 2009.

[18] R. Stanley, Enumerative Combinatorics, Vol. 2. Cambridge University Press, Cambridge, 1999.

[19] L. L. M. Yang, Generalizations of Han's Hook Length Identities. Online availaible at arXiv:0805.0109.

Markus Kuba, Institut für Diskrete Mathematik und Geometrie, Technische Universität Wien, Wiedner Hauptstr. 8-10/104, 1040 Wien - HTL Wien 5 Spengergasse, Spengergasse 20, 1050 Wien, Austria

E-mail address: kuba@dmg.tuwien.ac.at

Alois Panholzer, Institut für Diskrete Mathematik und Geometrie, Technische Universität Wien, Wiedner Hauptstr. 8-10/104, 1040 Wien, Austria

E-mail address: Alois.Panholzer@tuwien.ac.at 